\title{
SIBLING SPECIES IN THE EURYDICE GROUP OF LETHE (LEPIDOPTERA: SATYRIDAE)
}

\author{
By Ring T. Cardé, ${ }^{1}$ Arthur M. Shapiro, ${ }^{1}$ \\ and Harry K. Clench ${ }^{2}$
}

\section{INTRODUCTION}

We have uncovered a pair of sibling species in the comparatively well-known butterfly fauna of eastern North America: the common Grass Nymph or Eyed Brown, Lethe eurydice of recent authors, is actually two species, which are extensively sympatric.

Curiously, the distinctness of these two has been known since at least 1936, when W. D. Field discovered and characterized them as subspecies. He assigned names to them which we now know to be inapplicable. This was corrected in 1947 by R. L. Chermock, who named the presumably more southern "subspecies" appalachia. Neither of these authors was aware that the "subspecies" are sympatric.

The present investigation was first suggested when one of us (Clench) found both forms flying in the same area near Leesburg, Mercer Co., Pennsylvania in 1966. The conspicuous habitat difference between them implied that two species might be involved. In I968 another of us (Shapiro) found the same situation in western and central New York and (with Cardé) investigated the immature stages and biology of the insects. The results of this study are partly reported elsewhere (Shapiro and Cardé, 1970).

Independently of us, C. F. dos Passos and his correspondents simultaneously made the same discovery. Several of the conclusions contained in the resulting paper (dos Passos, 1969) appear erroneous. Since the taxonomic situation is very complex, we here review the whole subject, nomenclatorially, morphologically, and distributionally.

In brief, we recognize two species in this group, as follows:

( Ia) Lethe eurydice eurydice (Johansson), widely distributed from Labrador to Great Slave Lake and south to Delaware and Illinois, occurring in open marshes and sedge meadows.

( $\mathrm{Ib}$ ) Lethe eurydice fumosa (Leussler), scattered in small isolated colonies (many now extinct) in sedgy permanent marshes in the

\footnotetext{
${ }^{1}$ Department of Entomology and Limnology, Cornell University, Ithaca, N.Y. 14850.

${ }^{2}$ Section of Insects and Spiders, Carnegie Museum, Pittsburgh, Pa. 15213

*Manuscript received by the editor April 24, 1970.
} 


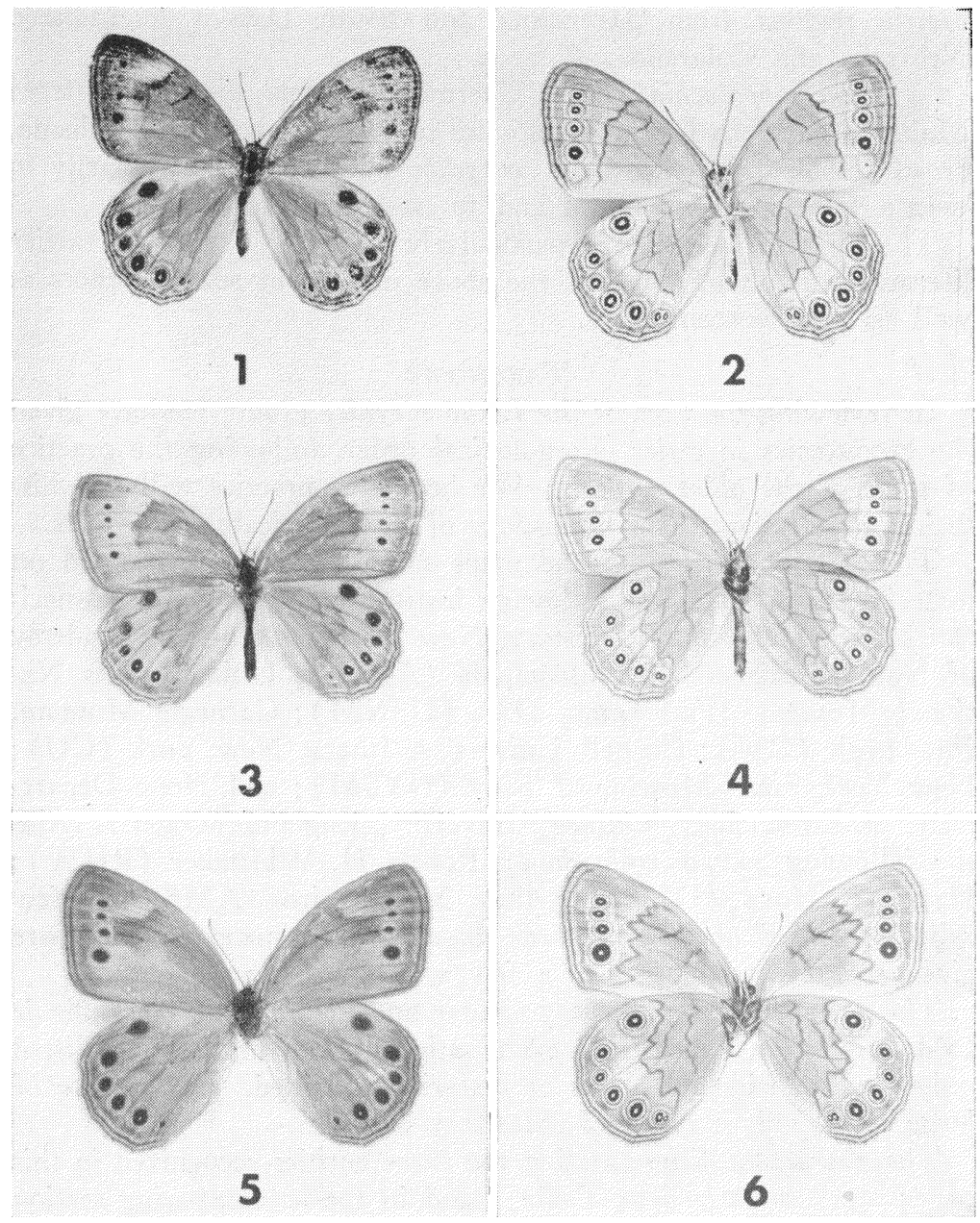

Figs. 1-2. Lethe appalachia male, McLean Bogs Reserve, Tompkins Co., New York. Figs. 3-4. Lethe eurydice (male) neotype, Morris Arboretum, Philadelphia Co., Pennsylvania. Figs. 5-6. Lethe eurydice fumosa male, Sarpy Co., Nebraska. 
prairie regions from Minnesota and South Dakota to Indiana, Nebraska and Colorado.

(2) Lethe appalachia R. L. Chermock, widely distributed from Maine to northern Florida, westward to South Dakota and Alabama, broadly sympatric with $L$. e. eurydice but occurring primarily in swamp forest, shrub swamps and forest-edge ecotones.

These forms have had a particularly extensive history in earlier literature, involving not just the above names but several others as well, as may be seen below.

\section{TAXONOMY}

In reviewing the taxa of the Lethe eurydice group, we have given the synonymies in strict chronological order, following the practice of some of the older authors. We hope this practice will add historical perspective to the discussions of these intricate synonymies.

The distributional data and maps (figs. 2I, 22) were based on material examined in the following institutional collections: American Museum of Natural History, New York (AMNH) ; Academy of Natural Sciences of Philadelphia (ANSP); United States National Musuem, Washington, D.C. (USNM); Carnegie Museum, Pittsburgh (CM); Cornell University, Ithaca, New York (CU); New York State Museum, Albany (NYSM); and Hope Department of Entomology, Oxford University, England (Oxon.). Also the following private collections: Robert $\mathrm{H}$. Whittaker (RHW); David J. Horn (DJH); Arthur M. Shapiro (AMS). A few reliable printed or other records, based on specimens not seen, are given separately, along with a few which are queried.

The sexes of Lethe are very similar and may at times be difficult to determine in a superficial examination. We have therefore listed only the localities and dates of material examined. In the case of large series, only a range of dates may be given.

The characters differentiating the three entities recognized in this paper are summarized in Table $\mathrm{I}$ and in the accompanying section of the text. A brief summary of the most conspicuous characters of each is given following its taxonomic history.

\section{Lethe eurydice eurydice (Johansson)}

Papilio eurydice Johansson 1763, Amoen. Acad. 6: 406; type locality Philadelphia [Pennsylvania]; type formerly in the De Geer collection (Stockholm, Sweden), now lost; neotype designated below.

Papilio canthus Linnaeus 1767 , Syst. Nat. (12th ed.): 768 ; type locality "in America septentrionali;" no type exists (replacement name for Papilio eurydice). 
? Papilio canthus: Fabricius 1775 (partim), Syst. Ent.: 486.

Argus canthus: Scopoli 1777, Introd. Hist. Nat.: 432.

Satyrus canthus: Godart 1821, Encycl. Meth. 9: 465, 493.

Neonympha canthus: Westwood 1851, in Doubleday, Westwood, and Hewitson, Gen. Diurn. Lep. 2: 375.

Neonympha cantheus (nec Godart 1821, see below): Morris 1860, Cat. Lepid. N. Amer.: 10.

Hipparchia boisduvallii Harris 1862, Ins. Inj. Veg. (Flint ed.) : 305, fig. 128; type locality "this State" (Massachusetts), type now lost; no neotype designated.

Debis canthus: Herrich-Schaeffer 1865, Correspbl. Zool.-Min. Ver. Regensburg 19: 72 .

Pararge canthus: Butler 1868, Cat. Satyridae Br. Mus.: 123.

Euptychia canthus: Kirby 1871, Syn. Cat. Diurn. Lep.: 55.

Pararge boisduvallii: Edwards 1872, Synopsis N. Amer. Butt.: 26.

Argus eurydice: Scudder 1872, Syst. Rev. Amer. Butt.: 6.

Satyrodes eurydice: Scudder 1875, Bull. Buffalo Soc. Nat. Sci. 2: 243.

Satyrodes canthus: Smith 1884, Bull. Brooklyn Ent. Soc. 6: 119.

? Satyrodes canthus ab. boweri F. H. Chermock 1927, Bull. Brooklyn Ent. Soc. 22: 118; type locality Port Hope, Ontario; type not located, stated by dos Passos to be in Carnegie Museum, but not found. (Infrasubspecific name with no standing in nomenclature.)

Satyrodes eurydice transmontana Field 1936, J. Ent. Zool. (Pomona) 28: 22 ; type locality Compton, Quebec; no type designated.

Satyrodes eurydice transmontana f. ㅇ rawsoni Field 1936, J. Ent. Zool. (Pomona) 28: 22; type locality Bloomfield, Michigan; type deposited in U. S. National Museum. (Infrasubspecific name with no standing in nomenclature.)

Lethe (Enodia) eurydice: R. L. Chermock 1947, Ent. News 58: 29.

The descriptions of both eurydice Johansson and canthus Linnaeus are too scanty to restrict on internal evidence to either of the sympatric northeastern species, both of which occur at the type locality (Shapiro, I970a). If that locality (Philadelphia) is accurate, there can be no doubt that Johansson's description applies only to a member of this group, even though no mention is made of eyespots on the forewing above (an objection to this usage, raised by Harris, I 862 and Edwards, I897). No type of eurydice or canthus (which was proposed explicitly as a replacement name for eurydice and hence has the same type) exists in the British Museum (Natural History) or in the De Geer collection at the Naturhistoriska Riksmuseum, Stockholm.

When appalachia (see below) was described as the southern subspecies of eurydice, the latter name became firmly associated with the assumed "northern" subspecies whose color and pattern were contrasted with appalachia by Chermock. It seems desirable to 
stabilize the nomenclature by preserving this usage through a neotype designation. This removes the possibility that a specimen of Chermock's appalachia might eventually be selected as neotype of eurydice, leaving the familiar "northern" insect's name in question. The only Philadelphia specimens of eurydice auct. with full data which we have found were collected by one of us (Shapiro). Several of these were placed in the United States National Museum two years ago, and we desiginate one such specimen the neotype of Papilio eurydice Johansson.

Neotype. - A male deposited in the U.S. National Museum bearing the manuscript label "eurydice $\sigma^{\top} /$ Morris Arboretum/Phila. Co. Pa./29 June I967/A. M. Shapiro" (fig. I). We have added a label identifying the specimen as the neotype of Papilio eurydice Johansson. The U.S.N.M. also contains a second specimen with the same locality and collection date.

Taxonomic History: the Euptychia names. - The taxonomy of L. eurydice is complicated by confusion with Yphthimoides (= Euptychia) argulus (Godart). This problem was not noted by dos Passos, and is reviewed here.

Fabricius (I775) reworked the description of canthus, adding "immaculatis" to the upperside diagnosis and altering various details. The "immaculatis" may have been by inference from the lack of reference to spots in the earlier descriptions, but it seems more likely that Fabricius was working from some other insect he confused with the Linnean one. In I 779 Cramer described and figured a species from Surinam as Papilio argante. This name is a junior homonym of Papilio argante Fabricius 1775 (now Phoebis argante, Pieridae). Fabricius synonymized argante Cramer to canthus (Fabricius, I78I), improperly emending it to arganthe in synonymy. (Arganthe is not available as a replacement name because it was proposed in synonymy.) He repeated this usage in 1787 and $\mathrm{I} 793$. His own descriptions of "canthus" do not fit Cramer's figure well. Godart (I82I) recognized that three species were included in the Fabrician concept "canthus" and attempted to end the confusion by redescribing the true canthus (translating Linnaeus), and naming two new entities, argulus and cantheus. Godart's argulus is a replacement name for the preoccupied argante and is the oldest valid name for this taxon. Cantheus is a renaming of the entity Fabricius first thought was canthus, theretofore without a valid name. The identity of this animal cannot be determined if, as appears, Fabrician specimens of "canthus" do not exist. 


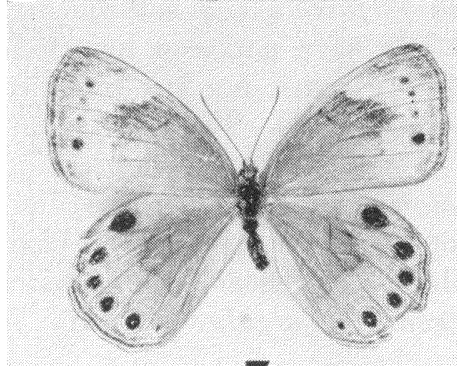

7

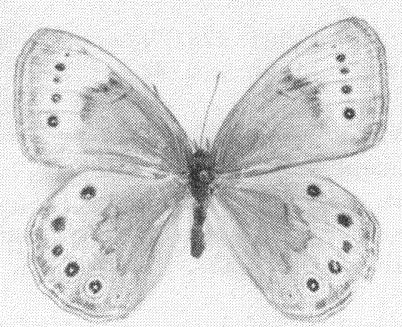

9

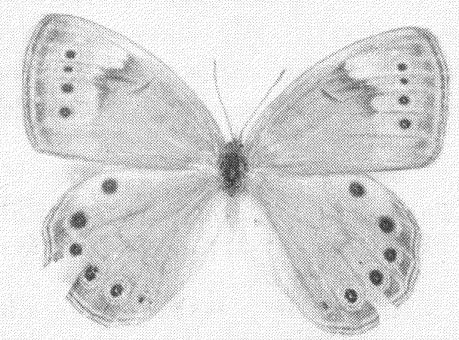

11

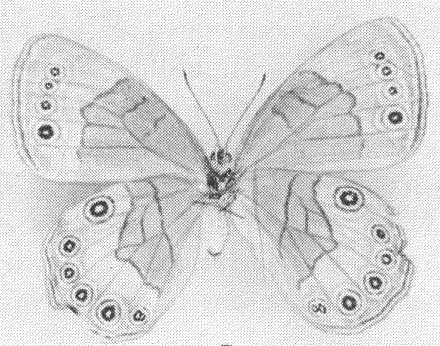

8

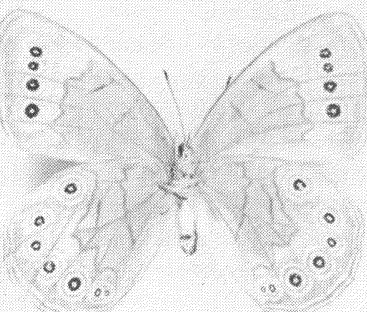

10

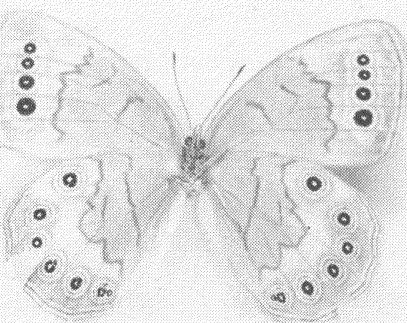

12

Figs. 7-8. Lethe appalachia female, McLean Bogs Reserve, Tompkins Co., New York. Figs. 9-10. Lethe eurydice eurydice female, McLean Bogs Reserve, Tompkins Co., New York. Figs. 11-12. Lethe eurydice fumosa female, Sarpy Co., Nebraska. 
Butler ( 1868) described Euptychia perfuscata and subsequently ( I869) synonymized it to argante. We have not seen his specimens (which should be in the British Museum), nor any specimen definitely determinable under any of these names. The most recent discussion of argulus is by Weymer (1907, p. 202). The leading North American authority on Euptychia and its allies, L. D. Miller, advises us (in litt.) that he does not know the species, but from Cramer's description and figure would place it near Yphthimoidies grimon. The partial synonymy of argante $=$ argulus is thus:

Papilio argante Cramer 1779 (nec Papilio argante Fabricius 1775), De Uitlandsch. Kapell. 3: 19, pl. 204; type locality Surinam; type not investigated.

\$ Papilio canthus (nec Linnaeus 1767): Fabricius 1781 (partim), Siec. Ins. 2: 64 (arganthe in synonymy); 1787, Mant. Ins. 2: 31; 1793, Ent. Syst. 3(1): 157 .

Satyrus argulus Godart 1821, Encyl. Meth. 9: 463, 488; type locality Surinam; type never existed (replacement name for argante).

? Euptychia perfuscata Butler 1868, Cat. Satyridae Br. Mus.: 18; type locality Para, Brazil; type probably in British Museum, not investigated.

? Euptychia argante: Butler 1869, Cat. Diurn. Lep. Fabr. Br. Mus. 13.

Cantheus, which is the unknown animal Fabricius confounded first with canthus and then with argante, usually appears in the synonymy of eurydice $=$ canthus, but its only proper claim there is its mistaken use in synonymy by Morris (1860). We have removed cantheus Godart from the synonymies of the other entities and regard it as a nomen dubium, presumably a species of Euptychia sens. lat. Its synonymy is:

† Papilio canthus (nec Linnaeus 1767): Fabricius 1775 (partim), Syst. Ent.: 486; 1781, Spec. Ins. 2: 64; 1787, Mant. Ins. 2: 31; 1793, Ent. Syst. $3(1): 157$.

Satyrus cantheus Godart 1821, Encyl. Meth. 9: 465, 493; type locality "l'Amerique septentrionale"; type not investigated, probably never existed.

Godart's description of cantheus erroneously cites Fabricius, "Species Insectorum" for "Mantissa Insectorum." The name is misspelled "cautheus" in the heading on page 465.

Taxonomic History: other names. - Gosse (I84I) attributes the name Hipparchia transmontana to Say, but it does not appear in any extant work by that author. It usually appears in the synonymy of eurydice attributed to Gosse I840. Apparently Gosse believed it was described elsewhere, since his "description" ( $1840, p .247)$ is 

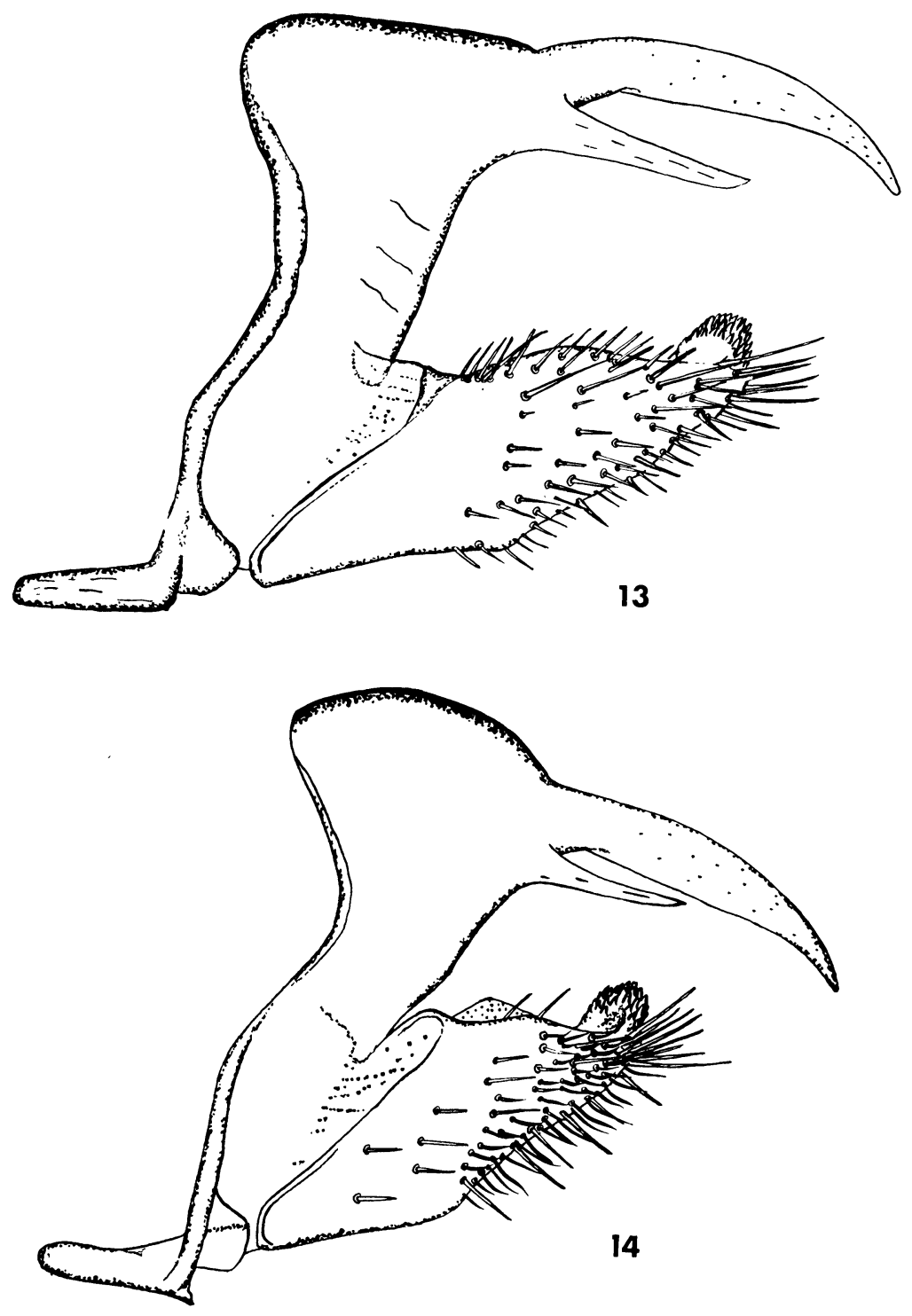

Fig. 13. Male genitalia of Lethe appalachia.

Fig. 14. Male genitalia of Lethe eurydice eurydice. 
inadequate to associate it with any biological entity. It could apply to any of several Quebec Satyridae, which are not exhausted by the other species enumerated in the text. His statement that "this is likewise described by the American naturalists as very rare, and is found only beyond the Rocky Mountains ..." makes no sense when applied to any species of Lethe. We regard this as a nomen nudum and have omitted it from the synonymy.

Field (1936) resurrected transmontana as the northern subspecies of eurydice, describing it adequately and giving as the type locality Gosse's base at Compton, Quebec. This is the oldest valid publication of the name, which should thus be credited to Field 1936. This subspecific distinction was grounded in confusion over the entities now called eurydice and appalachia. Observing differences between northern eurydice and specimens from near the type locality, Philadelphia, which he took as typical of that taxon but which were really appalachia, Field felt that a subspecific name was warranted. This is clear from his article, particularly the citation of Clark's ( I932) figures of Beltsville, Maryland appalachia which Field (like Clark) calls typical eurydice. Thus transmontana becomes a junior subjective synonym of eurydice. We can see no subspecific differences among eastern populations of eurydice as here restricted. Field's female form rawsoni is based on specimens faded in life; such specimens occur throughout the range of eurydice. The name is infrasubspecific and therefore has no formal standing.

The name boisduvallii was attributed by dos Passos (1964) to Morris (i 862), an error corrected later (dos Passos, I969). Morris published the name in synonymy, spelled boisduvalli. The first valid publication was in the posthumous ( I 862) edition of Harris's "Insects Injurious to Vegetation," edited by Flint. The editor's preface makes clear that the name should be attributed to Harris. It was emended to boisduvalii by Scudder ( 1889 ) in synonymy; this spelling is used by Forbes (1960) and dos Passos (1964). Dos Passos (1969) has further emended it to boisduvali. Although not the preferred form, the double " $i$ " is acceptable in taxonomy as the genitive of the Latinized name, i.e. "Boisduvalius." While Boisduval spelled his name with only one "l" and there is no orthographic reason to double it in forming the Latin genitive, the fact that the

Fig. 15. Male genitalia of Lethe eurydice fumosa (valve in slightly different position than valves of figs. 13 and 14). Fig. 16. Ventral view of Lethe appalachia valve. Fig. 17. Ventral view of Lethe eurydice eurydice valve. Fig. 18. Ventral view of Lethe eurydice fumosa valve. 

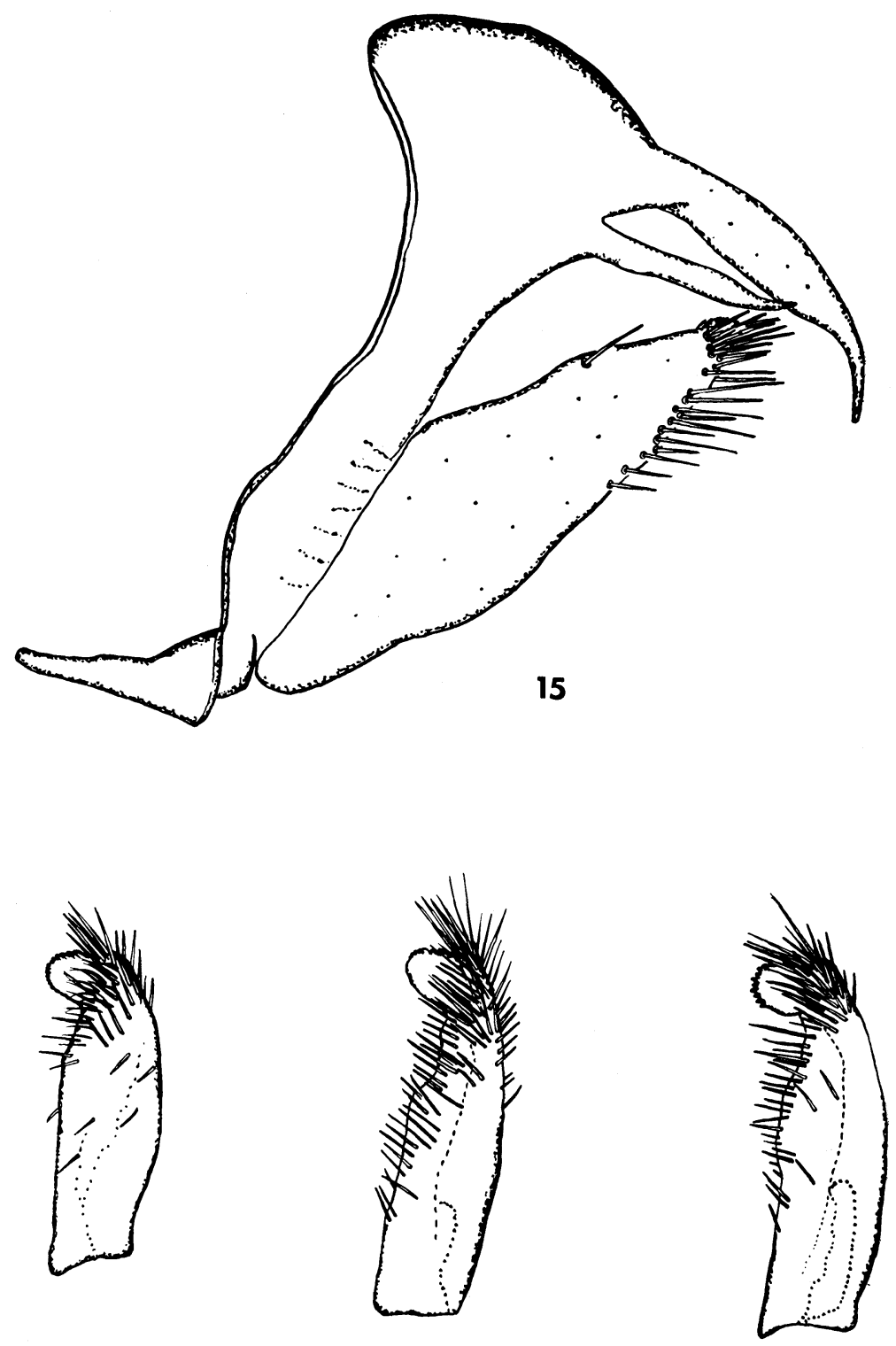

16 
name is spelled boisduvallii in both the text and index of Harris ( 1862 ) indicates that the double "l" was the form used in the Harris manuscript, and this is confirmed by its use in Morris's (I862) citation from that manuscript. Unfortunately, then, boisduvalli is technically a "correct original spelling" and cannot be emended under the Code.

Summary of Characters. - Lethe eurydice eurydice is relatively pale, pinkish brown above, somewhat yellowish beneath, with the postmedial line deeply indented beneath on all wings. The male valve is strongly 4 -sided when viewed laterally, and the tegumen is dorsally rounded. The larva appears superficially to have dark sidestripes on the head capsule, extending from near the tip of the horns to the ocelli. These and other characters are discussed more completely under the headings "Diagnostic Characters" and "Biological Differences," after the taxonomic treatment of Lethe appalachia, below.

Distribution (fig. 2I). - Material examined:

Delaware: New Castle Co.: Blackbird, vii.62-65 (ams); Kent Co.: Smyrna, vii.62-65 (AMs)

Pennsylvania: Philadelphia Co.: Morris Arboretum, vi.29.67 (AMS) (USNM), Tinicum Wildlife Preserve, vi-viii.6o-68 (AMS), George's Hill, no date (P. Laurent) (ANSP); Montgomery Co.: Cheltenham Twp., Horsham Twp., Montgomeryville, vi-viii.58-68 (AMs), Pennsburg, vi.2I-66 (aMs) (USNM) ; Bucks Co.: Buckingham, East Rockhill, Ivyland, Chalfont, Trevose, vi-viii.58-68 (AMS), Bristol, vi.27.67 (AMS) (USNM); Chester Co.: vic. Downingtown, vi-viii.58-68 (AMs); Susquehanna Co.: vii.22-31.? (CM); Lackawanna Co: Scranton, vii.4.05 (M. Rothke) (cM); Elk Co.: Medix Run, vii.I6.64 (H. K. Clench) (CM); Beaver Co.: New Brighton, vii.?.o3 (W. C. Wood coll.) (АMNH); Mercer Co.: 2 mi. SE Leesburg, vii.r. 66 (H. K. Clench) (cM), North Liberty, viii.3.6o, viii.9.59 (J. Bauer) (cM); Allegheny Co.: Nadine, vii.29.24 (cm); Erie Co.: Presque Isle, no date, vii.7.26, vii.?.4O $(\mathrm{cm})$

NEW Jersey: Camden Co.: Westville, Haddon Heights, Atco, Magnolia, vi-viii.58-68 (AMs) ; Burlington Co.: Mt. Holly, Whitesbog, vi-viii.58-68 (AMs); Gloucester Co.: Woodbury, Glassboro, vi-viii.58-68 (AMS) ; Atlantic Co.: Da Costa, vi-viii.58-68 (AMS); Mercer Co.: Pennington, Washington's Crossing State Park, Dutch Neck, vi-viii.58-68 (AMS); Ocean Co.: Lakehurst, New Egypt, viviii.58-68 (AMs); Middlesex Co.: Jamesburg, vii.8.32 (A.S. Pin- 
kus) (АмNH); Union Co.: Elizabeth, vii.r3.? (O. Buchholz) (AMNH); Sussex Co.: Arlington, vii.r3.18 (O. Buchholz) (AMNH), Hopatcong, no date (C. Palm) (AMNh), "Sussex Co." vii.I.43, vii.6.4I (O. Buchholz) (AMNH).

NEW YORK: New York Co.: West Farms, no date (J. Angus) $(\mathrm{AMNH})$; Queens Co.: Flushing, vii.27.I8 (E. L. Bell) (AMNH); Kings Co.: East New York, vii.?.o3 (W. C. Wood) (АMNH); Suffolk Co.: Calverton, v.26.25, vii.I4.29 (R. Latham) (cU), Orient, vi.2.38 (R. Latham) (cu) ; Richmond Co.: Staten Island, no date (Barnes coll.) (USNM), "S.I." no date (USNM); Rockland Co.: Spring Valley, vii.2o.68 (E. L. Rittershausen) (AMS); Orange Co.: I mi. E Monroe, vii.2 I.68 (E. L. Rittershausen) (AMS); Westchester Co.: Somers, no date, viii.9.16, vii.31.26 (W. C. Wood) (AMNH), Bedford, no date (R. B. Dominick), vii.I7.37 (A. C. Frederick), vii.r6-I8.37 (all AMNH), Lake Wacabuc, vii.I4.Io (AMNH); Sullivan Co.: Lava, vi.?.? (Barnes coll.) (USNM); Albany Co.: Karner, vii.rı.o3 (J. Cook) (Oxon.), Albany, vii.24.27, vii.25.32 (A. C. Frederick) (AMNH), vii.7.28 (A. C. Frederick) (cU); Otsego Co.: Cooperstown, vii.27.24 (B. Smith) (cu); Cortland Co.: $2.7 \mathrm{mi}$. W Willet, viii.2.68 (AMs), McGraw, vi.8.I4 (Engel coll.) (cM); Tompkins Co.: McLean, vic. Tompkins Co. Airport, Cayuga Inlet Valley, Michigan Hollow, Ringwood Hollow, Wilseyville, vi-ix.67-69 (AMS); Schuyler Co.: Texas Hollow, vii-viii.68 (AMs), Watkins Glen, vii.rg-68 (AMS); Yates Co.: Potter Swamp, vi.I4.I5 (cu); Oswego Co.: Minetto, vi.22.38 (W. T. M. Forbes) (cu); Livingston Co.: Lakeville, vii.r 8.27 (E. A. Maynard) (NYSM); Clinton Co.: Plattsburgh, vii.2.96, vii.r9.93 (G. H. Hudson) (NYSM) ; Columbia Co.: Ghent, viii.?.3 I ( $\mathrm{AMNH}$ ); Saratoga Co.: Saratoga Lake, vii.8.28 (A. C. Frederick) (AMNH); Jefferson Co.: Wellesley Island, viii.I3.68 (L. L. Pechuman) (AMs), Thousand Islands, vii.I2.09 (AMNH), Clayton, no date (J. H. Stebbins) (AMNH); Cattaraugus Co.: Crystal Lake, vii.6.3o (J. G. Franclemont) (cu); Erie Co.: Chafee, vi.I8.32 (J. G. Franclemont) (cu), Buffalo, no date (C. V. Riley coll.) (USNM) ; Lewis Co.: vii.I8.76 (W. W. Hill) (USNM), vii.r5.47 (C. P. Kimball) $(\mathrm{AMNH})$; Monroe Co.: vii.2.48, vii.23.46 (C. P. Kimball) (AMNH); Ontario Co.: Fishers, vii.30.48 (C. P. Kimball) (AMNH); Orleans Co.: Oak Orchard Swamp, vii.r6.68 (AMS); Genesee Co.: Oak Orchard Swamp, vii.ı6.68 (AMS); County undetermined: "NY," no date (G. D. Hulst coll.) $(A M N H)$, "vic. NYC" no date (S. L. Elliot) (AMNH) 
connecticut: Tolland Co.: Rockville, no date (Engel coll.) ( $\mathrm{CM}$ ) ; Litchfield Co.: Litchfield, vii.r.94, vii.r 5.94 (L. B. Woodruff) (AMNH); Windham Co.: Putnam, vii.2r.5O (A. B. Klots) $(A M N H)$; County undetermined: "Ct." no date (G. D. Hulst coll.) (AMNH)

MASSACHUSETTS: Worcester Co.: Winchendon, vii.3.? (J. A. Grossbeck) (AMH), Princeton, no date (W. T. M. Forbes) (cu); Middlesex Co.: Wayland, vii.7.21 (cu); Silver Hill, vic. Lincoln, vii.7.23 (figured by Clark, I932, pl. I, figs. 5, 6) ; County undetermined: "Mass." no date (Barnes Coll.) (USNM)

New hampshire: Cheshire Co.: Dublin, i899 (A. H. Thayer) (Oxon.), West Rindge, vii.r5.6o, vii.ıo.6r (DJH); Coos Co.: Jefferson, vii.r 5-2I.? (Engel coll.) ( $\mathrm{cm}$ ), vii.7.32 (G. \& J. Sperry) (AMNH), Shelburne, vii.4-IO.oI (USNM), White Mts., no date (H. Edwards) (AMNH); Grafton Co.: Franconia, no date (A. T. Slosson) (AMNH); Sullivan Co.: Claremont, Igo8 (USNM); County undetermined: "N.H." no date (H. Edwards coll.) (AMNH)

VERMONT: Windham Co.: Stratton, vii.2r.37 (H. Kahl) (cM); Rutland Co.: Mt. Killington, 4OOO', viii.I 7.4O (AMNH); County undetermined: vic. Sandgate, vii.r3.49 (A. B. Klots) (AMNH) MAINe: Piscataquis Co.: Greenville, vii.2 I-29.19 (F. Haimbach) (cM), Sebec Lake, vii.24-3ı.? (Barnes coll.) (USNM); Hancock Co.: Bar Harbor, vii.3.38 (A. E. Brower) (USNM), North Bluehill, vii.2r.23 ( $\left.A^{\prime} M N H\right)$, Mt. Desert, vii.?.? (W. C. Wood) (AMNH), vii.r3.33 (O. Buchholz) (AMNH); Kennebec Co.: Augusta, vii.23.38 (A. E. Brower) (USNM), vii.22.5o (A. E. Brower) (AMnh) ; Penobscot Co.: Orono, no date (M. Fernald) (cu), Bangor, no date (Engel coll.) ( $\mathrm{cm}$ ), vii.ro.89 (E. A. Smyth) (USNM), Passadumkeag Bog, vii.I-7.?' (W. Sweadner coll.) (CM), vii.r2.34 (A. E. Brower) (AMNH), vii.2.33 (L. P. Grey) (USNM), South Lincoln, vii.I5.5O (L. P. Grey) (АMNH), Lincoln, no date (L. P. Grey) (АMNH), (J. C. Hopfinger) (USNM), vii.ro.4O (J. C. Hopfinger coll.) (USNM) ; County undetermined: "Maine," no date (E. A. Smyth) (USNM)

oHIO: Stark Co.: Waynesburg, vii.2I.29, vii.4.3O (AMNH)

michigan: Allegan Co.: Douglas Lake, vii.ro.3o (H. C. Will) ( $\mathrm{CM})$; Livingston Co.: Pinckney, vii.9.? ( $\mathrm{cm}$ ), vii.9.39, vii.23.39 (Амnн), George Reserve, Pinckney, vii.23.38, vii.3I.38 (G. W. Rawson) (USNM), "Livingston Co." vii.9.32 (G. W. Rawson) (USNM) ; Branch Co.: no date (B. Stroup) (cU); Otsego Co.: 
Sturgeon River, 5 mi. E Vanderbilt, vii.8.55 (Klots \& Rindge) $(A M N H), 7$ mi. E Vanderbilt, vii.7.55 (F. H. Rindge) (AMNH), Lake Otsego, vii.7.55 (F. H. Rindge) ( $\mathrm{AMNH}$ ), Pigeon River, I I mi. E Vanderbilt, vii.8.55 (Klots \& Rindge) (AMNH); Cheboygan Co.: vii.6.52, vii.r 9.52 (H. V. Daly) (AMNH); Emmett Co.: 6 mi. W Pellston, vii.9.55 (Klots \& Rindge) ( $\mathrm{AMNH}$ ), Petoskey, vii.8.ı 3, vii.8.I4 (J. J. Lichter) (АмNн), Galloway Lake, North Levering, vii.9.55 (Klots \& Rindge) (AMNH); Huron Co.: Hume Twp. Arboretum, vi.28.52 (H. V. Daly) (AmNH); Schoolcraft Co.: Thompson, vii.ı.55 (Klots \& Rindge) ( AMNH); Oakland Co.: New Hudson, vi.20.27 (G. W. Rawson) (USNM), Bloomfield, viii.4.29 (G. W. Rawson) (AMNH), viii.r2.28 (G. W. Rawson, paratype of rawsoni Field) (USNM), viii.4.29 (G. W. Rawson) (USNM) ; Washtenaw Co.: Willis, vii.30.39 (AMNH), Sharon, vii.2.44. (G. W. Rawson) (USNM) ; County undetermined: "Michigan" vii.8.9o ( son) $(\operatorname{AMnh})$, Calvin, vii.3.90 ( АмNн), "Snow I., Lake Michigan," no date (cM)

INDIANA: Steuben Co.: vi.r6.o3 (AMNH); Lake Co.: Hessville, vii.4.08 (E. Beer) (USNM)

ILlinors: Lake Co.: "NE Lake Co." viii.24.3o (H. M. Bower) $($ AMnH) ; Cook Co.: Chicago, vii.6.r3 (J. D. Gunder coll.) (AMNH)

minnesota: Ramsey Co.: St. Paul, no date (Barnes coll.) (USNM); County undetermined: "Minn." no date (AMNH) Wisconsin: Douglas Co.: 2 mi. E Maple, vii.ri.55 (Klots \& Rindge) ( (H. M. Bower) (AMNH); Waukesha Co.: Dousman, vii.r4.I6, vii.2o.I9 (H. M Bower) (AMNH); Dane Co.: Madison, no date (E. T. Owen coll.) (USNM) ; County undetermined: "Wis." no date (A. T. Slosson) (amnh), (E. T. Owen coll.) (UsNm) nova scotia: Cape Breton, viii.?.49 (G. Macmillan) (см); Cape Breton National Park, viii.?.54 (H. Dietrich) (cU)

NEW BRUNSWICK: Bathurst, viii.5-6.5 I (A. B. Klots) (AMNH) QUebec: Dunlop Rd., Gatineau Provincial Park, vii.6.52 (F. H. Rindge) (AMNH); Montreal, vii.r.29 (J. C. Hopfinger coll.) (USNM); "Quebec" vii.3.35 (J. C. Hopfinger coll.) (USNM) ontario: Ottawa, bred (W. H. Edwards) (cm), vii.23.97 (M. Holmes) (Oxon.); Toronto, viii.g.24 (R. N. \& F. A. Dixey) (Oxon.), vii.I9.I5, vii.20.I8 (H. V. Andrews) (cM); Bancroft, vii.I-7.? (W. Sweadner coll.) ( $\mathrm{cm})$; Spider Lake, Georgian Bay, 
vii.22.14 (G. K. Jennings) ( $\mathrm{cM}$ ) ; Point au Baril, vii.2 I.35 (E. D. McDonald) (cU) ; Don Valley, Toronto, vii.8.57 (J. C. E. Riotte) $($ AMNH $)$; Sudbury, vii.7-I0.59, vii.7-10.60 (J. C. E. Riotte) $(A M N H)$, vii.7.6o and vii.I2.58 at UV lights (J. C. E. Riotte) (АMNH); Gravenhurst, Muskoka Dist., iv.7.I8 (!) (АMNH); Geraldton, Ashmore Twp., vii.r6.55 (Klots \& Rindge) (AMNH); Grand la Cloche, vi.27.4I, vii.I.4I (O. Buchholz) (AMNH); Leamington, Essex Co., vi.?.9o (E. A. Smyth coll.) (UsNm) ; "Ont." no date (Blackmore coll.) (USNM)

manitoba: Aweme, vii.r 5.07 (cM), vii.I9.o8 (Barnes coll.) (USNM) ; Riding Mts., vii.r I.38, vii.9.39, vii. 2-3.40 (C. S. Quelch) $($ AMNH), vii.I7.38 (J. F. May) ( son) (UsNw); Transcona, vii.I9.48 (C. S. Quelch) (AMNH); Birtle, vii.7.44, vii.r9.44 (J. Dennis) (AMNH); Telford, Whiteshell Provincial Park, vii.24.55 (Klots \& Rindge) (AMNH)

ALbERTA: Rivercourse, near Lloydminster, vii.6.4I (R. J. Fitch) $(\mathrm{CU})$

Other records: Dos Passos (I969) erroneously records eurydice from "south to Colorado and east of the Rocky Mountains to Georgia and Florida." The Colorado records represent L. e. fumosa (see below). At present the southernmost record of true L. e. eurydice is northern Delaware.

Scudder ( I889) records L. eurydice from Rupert's Fort, Quebec (east shore Hudson's Bay); Mingan, Labrador; and Great Slave Lake, NWT. All of these are plotted on the map.

The western distribution of eurydice is unclear. Puckering and Post ( I960) record it from Cass, Cavalier, Dickey, Grand Forks, and Pembina Cos., North Dakota. These are entered on the map. We have seen no South Dakota records. However, Leussler ( 1938 ), who was well acquainted with $L$. e. fumosa, reported typical eurydice in Sioux Co., northwestern Nebraska. This record seems to require special confirmation, and has not been plotted.

The "eurydice" reported from Monroe Co., Tennessee (Mather, I96I) was appalachia (W. Reinthal, pers. comm.). The latter species was found at Jackson, Tenn. by Roever (Mather and Mather, I958).

\section{Lethe eurydice fumosa (Leussler)}

Satyrodes canthus n.v. fumosus Leussler 1916, Ent. News 27: 99, pl. iv, figs. 1,2 ; type locality Sarpy County, Nebraska; type reportedly deposited in Ohio State University, not seen.

Lethe fumosus: dos Passos 1969 (partim), J. New York Ent. Soc. 77: 120. 
Taxonomic History. - Described from 17 males and 8 females, all labeled "Omaha" by Leussler, as are various later topotypes. Although it was described as a "variety," the geographic nature of fumosa was clearly expressed.

The Greek noun $\lambda \dot{\eta} \theta \eta$ is feminine and retains its gender in the Latinized form Lethe. Both the species names in this group are also feminine. We have adopted the spelling fumosa to make this subspecies agree in gender, as provided by the Code.

Summary of Characters. - Lethe eurydice fumosa resembles L.e. eurydice in most respects, but the males and some females are darker above. The four eyespots on the forewing are consistently graded in size, from the smallest on top to the largest at the bottom; this is especially obvious beneath. The male valves have far fewer setae than in $L . e$. eurydice. The early stages are unknown.

Distribution (fig. 2I ). - Material examined:

NeBraska: Sarpy Co.: "Omaha" vi.28.13, vii.r.I5 (R. A. Leussler) (cotypes) (ANSP); vii.I2.13, vi.r4.13, vi.27.I4 (cotype), vii.7.I7 (R. A. Leussler) (USNM) ; vi.27.I4 ("topotype"), vii.5.I3, vi.28.13, vi.26.I5 (cotype), vii.7.I7 (R. A. Leussler) (AMNH); vi.27.14 (paratype), vii.r.I6 (figured by Holland, pl. 63, fig. II) (R. A. Leussler) (cM) ; County undetermined: "Nebraska," no date (J. Angus coll.) ( $\mathrm{AMNH})$

IowA: Dickinson Co.: Lake Okoboji, vi.25.2I (R. A. Leussler) (USNM) ; Hancock Co.: I mi. W Klemme, vii.24.6o (L. D. Miller) ( $\mathrm{CM})$; Powesheik Co.: Grinnell, vii.4.8 I (AMNH) ; County undetermined: "Ia." no date (H. Skinner) (cM)

wisconsin: Kenosha Co.: Twin Lakes, vi.I-4.I I (A. Kwiat) (USNM); County undetermined: "Wis." no date (E. T. Owen coll.) (USNM)

minnesota: Hennepin Co.: Lake Minnetonka, no date (USNM), St. Anthony Park, vii.r 5.9I (USNM)

Indiana: County undetermined: Tremont, vii.24.48 (O. Buchholz) (AMNH)

South Dakota: Brookings Co.: Volga, no date (P. C. Truman) $(\mathrm{CM})$

COLORADo: Larimer Co.: Loveland, no date (W. H. Edwards coll.) ( $\mathrm{CM}$ ); County undetermined: "Colo." not date (David Bruce) (cm), "Colorado" no date (E. T. Owen coll.) (USNM), "Colorado" no date (H. S. Burnett) (UsNM) 
Bruce collected fumosa at Estes Park, Larimer Co., Colorado (Edwards I897, p. 197). The identities of the Indiana, Minnesota, and Wisconsin specimens noted above were not checked by the genitalia, and are somewhat uncertain.

\section{Lethe appalachia R. L. Chermock}

Lethe (Enodia) eurydice appalachia R. L. Chermock 1947, Ent. News 58 : 29; type locality Conestee Falls, North Carolina; type in R. L. Chermock collection, not seen.

Lethe fumosus appalachia: dos Passos 1969, J. New York Ent. Soc. 77 : 121.

Taxonomic History. - Unmistakable figures of appalachia appear in three older works under other names. None of these has any taxonomic significance. Boisduval and Le Conte (1829) figure a male appalachia with an ambiguous female as Satyrus canthus (pl. 60). Edwards (1897) figures a female appalachia (pl. 26, figs. 3, 4) with a normal male eurydice (figs. I, 2) as Satyrodes canthus, along with a dark male which is probably also eurydice but might be fumosa (fig. 5). Denton (1900) figures an ambiguous specimen (p. 217), an eurydice (p. 218), and an appalachia (p. 219), all as Neonympha canthus.

Dos Passos (I969) erred in sinking appalachia to "fumosus." There is no evidence for his statement that "fumosus and appalachia occur at opposite ends of a cline."

Dos Passos lists ab. boweri F. H. Chermock under appalachia. It cannot be identified to species by the description, and the type has not been found; it is not in the Carnegie Museum, where dos Passos recorded it. We have placed boweri provisionally in the synonymy of eurydice because that species is considered more likely from the type locality, Port Hope, Ontario. However, a specimen of appalachia with no ocelli on the forewings above, labeled "Bowie, Md./ v-29-45/DDT experiment," is in the U.S. National Museum. At any rate, the name is clearly infrasubspecific and has no standing.

Summary of Characters. - Lethe appalachia differs from both subspecies of eurydice in being grayish or mousy brown above (blackish when fresh) and somewhat purplish or lilac-tinged beneath; the postmedial lines rounded, with only slight indentations. The male valve is less clearly 4 -sided in lateral view, and the tegumen is dorsally flattened. The larval head capsule bears side-stripes not reaching below the bases of the horns.

Distribution (fig. 22). - Material examined:

FLORIDA: Jefferson Co.: Monticello, x.4.I4 (paratype) (АMNH) 


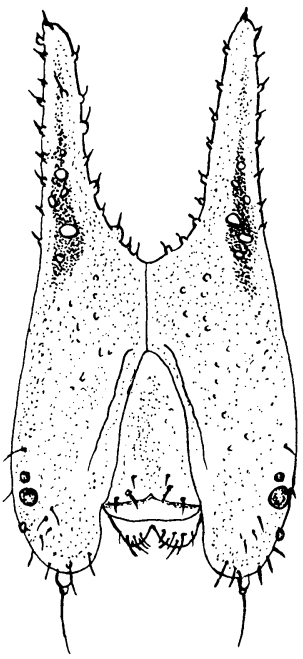

19

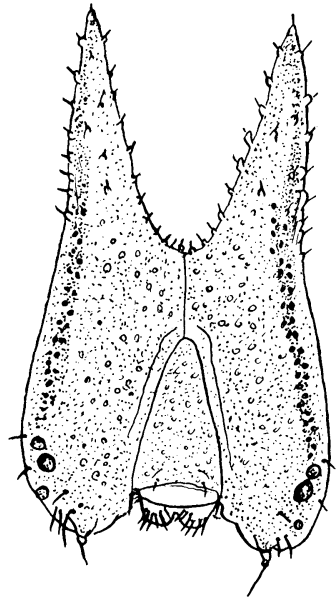

20

Fig. 19. Larval head capsule of Lethe appalachia.

Fig. 20. Larval head capsule of Lethe eurydice eurydice.

SOUTh CARolina: Jasper Co.: Coosawhatchie, vii.26.36 (R. B. Dominick) (paratype) (AMNH) ; Clarendon Co.: ix.?.89 (E. A. Smyth) (USNM) (see Smyth, i890)

NORTh carolina: Mitchell Co.: vii.?.92 (O. Buchholz) (AMNH); Transylvania Co.: Conestee Falls, vii.I-7.?, vii.I5-2I.? (CM) virginia: Amherst Co.: i936 (J. Bauer) (cM); Prince George's Co.: New Bohemia Swamp, viii.22.67 (J. Bauer) ( $\mathrm{CM}$ ) ; Nansemond Co.: Dismal Swamp, vi.r9.4O (cM); Giles Co.: Little Meadows, vii.25-26.40 (L. Carr) (paratypes) (UsNm); Montgomery Co.: viii.20.98 (E. A. Smyth) (Usnm); Fairfax Co.: Vienna, vii.r 9.38 (A. H. Clark) (paratype) (USNM); Wythe Co.: Speedwell, viii.r I.38 (A. H. Clark) (paratype) (USNM); Grayson Co.: Long's Gap, viii.ı I.38 (A. H. Clark) (paratype) (UsNM); County undetermined: Glen Carlyn, viii.r2.? (A. N. Caudell) (paratype) (USNM)

DISTRICT OF COLUMBIA: Washington, vii.I7.29 (G. W. Rawson) (USNM), vi.29.29 (paratypes) (USNM), vi.I7.29 (paratype) (figured by Clark, I932, pl. I, figs. 3,4) (USNM)

MARYland: Prince George's Co.: Hyattsville, vi.2o.39 (G. W. 
(Rawson) (USNM), Bowie, v.29.45 (USNM), Beltsville, vii.r 5.28 (paratype) (USNM); Calvert Co.: Mutual, vi.21.36 (G. W. Rawson) (USNM)

Delaware: New Castle Co.: Christina, vii.64 (ams)

PENnsylvania: Crawford Co.: Hartstown, vii.4.2 I (H. Kahl) (CM), viii.8-I 4.? ( $\mathrm{cm}$ ); Mercer Co.: North Liberty, viii.3.6o (J. Bauer) (CM), 2 mi. SE Leesburg, vii.r r.66 (H. K. Clench) (CM); Butler Co.: Slippery Rock, vii.4.3I (W. Sweadner) (CM); Fayette Co.: Dunbar, vii.6.3I (cM) ; Westmoreland Co.: Powdermill Nature Reserve, vi-viii.56-68 (H. K. Clench) (cM); Chester Co.: Exton, vii-viii.59-68 (AMs); Montgomery Co.: Horsham Twp., Cheltenham Twp., Enfield, vi-viii.59-68 (ams); Delaware Co.: Chadd's Ford, vii-viii.62-66 (aMs); Philadelphia Co.: Mt. Airy, v.28.? (P. Laurent) (AMNH), Tinicum Wildlife Preserve, Eastwick, vii-viii.58-68 (AMS)

NeW Jersey: Cape May Co.: Woodbine, vi-viii.59-67 (AMs); Camden Co.: Westville, viii.6.92, viii.r 4.92 (P. Nell) (cM), vi.I2.? (P. Laurent) ( $\mathrm{CM})(\mathrm{AMNH})$; Burlington Co.: Warren Grove, Wading River, vii-viii.64-68 (AMS); Gloucester Co.: Wenonah, viii.2r.ı (CM); Ocean Co.: Cassville, viii.r7.ro ( $\mathrm{AMNH}$ ); Morris Co.: Green Village, vii.30.? (C. Rummel) (cM), vii. 5.? (C. Rummel) (ANSP), "Morris Co." vii.21.50 (P. Ehrlich) ( $\mathrm{AMNH})$, vii.9.30, vii.6.4I, vii.I8.5O (O. Buchholz) (AMNH); Union Co.: vi.r6.4O (O. Buchholz) (AMNH); Somerset Co.: Orange Mts., vi.I4.?, viii.2O.3I (O. Buchholz) (AMNH); Bergen Co.: Ramsey, viii.?.I7 (AMNH) ; Passaic Co.: Paterson, vii.r 7.? (J. A. Grossbeck) (AMNH); Sussex Co.: Springdale, vii.9.49 (P. Ehrlich) (АмNн), vii.Io.49 (N. W. Gillham) (АмNH), Lake Lackawanna, vii.9.49 (P. Ehrlich) (AMNH), Stanhope, vii.28.33 (C. Rummel) (AMNH), Hopatcong, no date (C. Palm) (АмNH), "Sussex Co." vii.9.30, vii.6.4I, vii.r.43, vii.r8.50 (O. Buchholz) $($ AMNH $)$; County undetermined: "N.J." (Neumoegen coll.) (USNM), "N.J." (C. Palm) (AMnh)

NEW YORK: New York Co.: West Farms, no date (J. Angus) (AMNH), Bronxville, vii.9.II, vii.22-23.II (L. B. Woodruff) $(\mathrm{AMNH})$, Bronx, bred (E. Gerstenkorn) (AMNH); Suffolk Co.: Riverhead, vii.7.49, vii.8.49, vii.I7.52, vii.5.53, viii.rI.53 (R. Latham) (cu), Orient, vi.30.4I, viii.2.49, vi.I7.52 (R. Latham) (cu), East Hampton, vi.r4.49 (R. Latham) (cu), Greenport, viii.I.20, ix.I.5I, ix.6.28 (R. Latham) (cU), Calverton, vii.8.30 (R. Latham) (CU), Brookhaven, vii.5-I3.65 (RHW); 
Queens Co.: Flushing, vii.27.18 (E. L. Bell) (AMNH); Westchester Co.: Bedford, vii.7-9.34, vii.r7.37 (R. B. Dominick) $(\operatorname{AMNH})$, Somers, no date (W. C. Wood) (AMNH), Crugers, vii.r6.I2 (AMNH); Dutchess Co.: Fishkill, ix.7.65 (DJH); Sullivan Co.: Lava, no date (Barnes coll.) (USNM); Albany Co.: Albany, vii.24.27 (A. C. Frederick) (AMNH), Karner, vii.7.7O (Lintner) (USNM), vii.20.79 (W. W. Hill) (NYSM), vii.r I.03 (J. Cook) (Oxon.) ; Tompkins Co.: McLean, vii.I 8.9 I (local collection, CU), viii.7.25, vii.27.29, viii.I.25 (cU), vii.I8-2I.68 (AMs), Sapsucker Woods, viii.7.68 (AMS); Schuyler Co.: Texas Hollow, viii.3.68 (AMs); Orleans Co.: Oak Orchard Swamp, vii.r6.68 (AMs) ; Genesee Co.: Batavia, vii.r6.87 (cu) ; Cattaraugus Co.: Allegany State Park, vii.2I.4O (A. R. Shadle) (USNM); Franklin Co.: Paul Smith's, vii.?.o3 (A. P. Hunt) (Oxon.); County undetermined: "New York" (H. Edwards coll.) (AMNH) connecticut: New Haven Co.: Sound View, vii.r6-21.34 (A. H. Clark) (USNM) ; Litchfield Co.: Sharon, vii.14-2I.4O, vii.?.4I (L. J. Sanford) (AMNH) (one figured by Klots, I95I, pl. IO); Fairfield Co.: Stamford, vii.22.37 (J. G. Thorndike) (AMNH); Hartford Co.: Avon, viii.r.03 (R. C. Williams) ( $\mathrm{CM}$ ), vii.ı 8.22 (R. C. Williams) (ANSP)

Massachusetts: Suffolk Co.: Newton Highlands, no date (W. Barnes) (USNM) ; Hampden Co.: Wilbraham, viii.?.94 (cU); Bristol Co.: Swansea, vii.r 8-22.34 (E. T. Learned) (ANSP) RHODE ISLAND: Providence, vii.IO-2O.? (H. Engel) ( $\mathrm{CM}$ ), North Scituate, I9I2 (G. H. \& J. L. Sperry) (AMNH)

New hampshire: Coos Co.: Jefferson, vii.7.32 (G. H. \& J. L. Sperry) (AMNH), "White Mts." no date (W. H. Edwards) (cm) MaIne: Penobscot Co.: Bangor, no date ( $\mathrm{CM})$

Quebec: "Quebec," vii.3.35 (J. C. Hopfinger coll.) (Usnm)

Illinors: Cook Co.: Chicago, vii.6.I3 (J. D. Gunder coll.) ( $\mathrm{AMNH})$

INDIANA: Lake Co.: Hessville, vii.4.o8 (E. Beer) (USNM); County undetermined: "Indiana," no date (E. A. Smyth coll.) (USNM)

Michigan: Huron Co.: Hume Twp. Arboretum, vi.28.52 (H.V. Daly) ( АMNH); Cass Co.: Wakelee, viii.3.58 (L. J. Sanford) $(\mathrm{AMNH})$; County undetermined: "Michigan," no date (AMNH) minnesota: Hennepin Co.: Lake Minnetonka, viii. ?.86 (АмNн) wisconsin: County undetermined: "Wis." no date (E. T. Owen coll.) (USNM) 
SOUTH DAKota: Brookings Co.: Volga, no date (Ehrman coll.) $(\mathrm{CM})$

MIssouri: St. Louis Co: St. Louis, vii.ro.? (CM)

We regard the South Dakota and Missouri records of L. appalachia as somewhat dubious, but they are shown on the map.

Other records: The following are probably accurate, although the specimens have not been seen. They are included on the map:

GEORgIA: Thomas Co.: Linton Lake, viii.9.67, viii.29.67; Fulton Co.: Atlanta (Harris Trail), vii.29.6o, viii.20-26.6I : De Kalb Co.: Avondale Estates, vi.r6.44; Union Co.: Copper Creek State Park, vi.6.58, vii.ı6.6ı, vi.ı 8.62, vii.ı8.62, viii.22.59; White Co.: Cleveland, vi.r3.57 (all from L. Harris, unpublished MS, p. 244)

Minnesota: Anoka Co.: Bald Eagle Lake, i966 (Masters, i967) Indiana: Steuben Co.: Hogback Lake, vii.I7.42 (Price and Shull, I 969)

Tennessee: Madison Co.: Jackson (Mather and Mather, I958) alabama: Tuscaloosa Co.: vic. Tuscaloosa (Chermock, I949) MICHIgan: Montcalm Co.: Sidney, vii.26.5o (F. Rutkowski) Pennsylvania: Fayette Co.: Markleysburg Bog, 2 mi. N Markleysburg (H. K. Clench)

\section{Diagnostic characters}

Color and Pattern.- Lethe e. eurydice and L. e. fumosa differ subtly but consistently from L. appalachia. Nearly all specimens can be assigned to the correct species by color and pattern alone. The most useful characters separating the two species are the ground color above and beneath, and the waviness of the postmedial line beneath. The two subspecies of eurydice differ most consistently in the relative sizes of the forewing ocelli. All of the observed differences are given in Table $\mathrm{I}$.

We have not seen a truly fresh specimen of $L$. $e$. fumosa. Leussler (1916) describes the ground color of fresh specimens as "a very dark smoky grey .. . even a blackish appearance." This sounds very much like the color of newly emerged appalachia. Old specimens of the two are very different, however: fumosa males are an even, somewhat purplish or reddish brown, while appalachia is grayish or mousy brown. Some female fumosa, particularly from Colorado, are nearly identical in color to nominate female eurydice, but the ground color of the males is nearly always distinctive. A few male eurydice from the northeast are dark purplish brown when 
fresh, and fade to an even dark reddish brown. Their spot-sizes are normal and they lack the fumosa tendencies to "high angledness" of the forewing and blind and rimless ocelli above. A specimen of this dark form of nominate eurydice is probably represented by Edwards' figure 5 ( $1897, \mathrm{pl} .26$ ).

Specimens of the three taxa are shown in figs. I-I2.

Male Genitalia. - Chermock (1947) and dos Passos (1969) reported no genitalic differences between $L$. e. eurydice and $L$. appalachia. However, we have found that they do differ slightly but significantly. The tegumen of appalachia is flattened dorsally, while that of eurydice (both subspecies) is rounded. The valves of appalachia are shorter and narrower dorso-ventrally, and from the side appear less quadrilateral than those of the eurydice subspecies. The male genitalia of $L$. e. eurydice and $L$. e. fumosa are substantially similar, but differ from each other and from appalachia in the density and arrangement of setae on the valves. See Table $I$ and figs. I3-I 8 .

Female Genitalia. - There seem to be no useful characters here. Some minor differences in the sclerotization of the genital plate were found among all three taxa.

Early Stages. - The larvae of L. e. eurydice and L. appalachia from central New York differ consistently in the maculation and tubercles of the head capsule. In $L$. e. eurydice the red side stripes become darker below the bases of the horns, extending to the ocelli. The darker part of the stripe consists of small, heavily pigmented, regularly arranged tubercles on a less heavily pigmented ground. In $L$. appalachia the stripe does not extend below the horn, and its lower end contains several large, pale, irregularly placed tubercles which contrast with the red ground (figs. 19, 20).

The early stages of $L$. e. fumosa are completely unknown.

\section{BIOLOGICAL DIFFERENCES}

Developmental Rate. - Larvae of L. e. eurydice and L. appalachia from McLean, N.Y. reared $e x$ ovo at $24^{\circ} \mathrm{C}$ on late summer photoperiods showed developmental differences. Eurydice larvae invariably entered diapause in the third or fourth instar. Appalachia larvae usually developed without diapause, the entire life cycle requiring about 60 days. Lethe appalachia is at least double-brooded in its southern range; apparently it has the potential to breed continuously 
Table 1. Differences separating taxa of the Lethe eurydice group. Color terminology follows Kornerup and Wanscher (1963) and Ridgway (1912).
character
L. e. eurydice

\section{COLOR AND PATTERN}

postmedial line of forewing beneath projects marginad into teeth at $\mathrm{M}_{3}$, $\mathrm{Cu}_{2}$

postmedial line of hindwing beneath projects marginad into teeth at $\mathrm{Cu}_{1}$, $\mathrm{Cu}_{2}$

ground color beneath (fresh males) red-haired $(6 \mathrm{C} 4)=$ wood brown

ground color beneath

greyish orange $(5 \mathrm{~B} 5)=$ clay color

(fresh females)

ground color above (fresh males) sunburn (6D5) $=$ snuff brown

ground color above (fresh females) clay (5D5) $=$ Saccardo's umber

color between ocelli and subterminal darker than ground, tinged with line beneath orange

color marginad of postmedial line lighter than ground, yellowish on hindwing beneath especially in $\mathrm{M}_{3}$

contrast between discal and limbal moderate to strong areas on forewing above

rings around ocelli above

usually strong

ocelli of forewing beneath

variable, subequal, 4 usually largest

ocelli 4,5 on hindwing above

usually pupilled

apex of forewing

tending to be "low angled"d

\section{MALE GENITALIA}

valve shape

4-sided in lateral view

valve costa

inner lip larger

valve setae

valve tip heavily armed;

many setae on sacculus

tegumen

dorsally rounded

III. LARVAL HEAD CAPSULE

red stripe

top of horns to ocelli

tubercles in red stripe

red, small, regularly arranged

IV. HABITAT

open sedge marshes

anly old specimens seen

${ }^{\mathrm{b}}$ fades in life to Van Dyke brown $(6 \mathrm{~F} 6)=$ bistre

${ }^{\mathrm{c}}$ fades in life to brown $(6 \mathrm{E} 5)=$ brownish olive 
as in L. e. eurydice

as in L. e. eurydice

cinnamon $(6 \mathrm{D} 6)=$ Rood's brown greyish orange $(5 \mathrm{~B} 5)=$ clay color

cocoa brown $(6 \mathrm{E} 6)=$ cinnamon brown $^{\mathrm{a}}$

sahara $(6 \mathrm{C} 5)=$ sayal brown ${ }^{\mathrm{a}}$

as in $L . e$. eurydice

as in $L . e$. eurydice

slight ( $\hat{o})$ to moderate (우)

weak to strong

usually $4>3>2>1$

frequently unpupilled

"high angled" in $\hat{\delta} \hat{\delta}$ only

as in L. e. eurydice

as in L. e. eurydice

few setae on valve tip or sacculus

as in L.e. eurydice

unknown

unknown

permanent marshes

within prairie region smoother, only slightly wavy

smoother, only slightly wavy

dark blonde (5D4) = buffy brown

topaz $(5 \mathrm{C} 5)=$ avellaneous

teak $(6 \mathrm{~F} 5)=$ mummy brown ${ }^{\mathrm{b}}$

teak $(6 \mathrm{~F} 5)=$ mummy brown ${ }^{\mathrm{c}}$

paler, not orange-tinted

lighter than ground, with violet iridescence

moderate to strong

weak to strong

usually 1 and 4 largest

frequently unpupilled

frequently "high angled" (both sexes)

less 4-sided; short; narrower dorso-ventrally inner lip smaller some setae distally and on sacculus

dorsally flattened

horns only

irregular, large, pale

swamp forest, shrub swamp, forestedge ecotones

\footnotetext{
d"high angled": ratio of length of forewing (base to apex):
} outer margin $<1.5$. 
(without diapause) elsewhere as well. So far as is known, eurydice is single-brooded everywhere. Larvae of both species turn from yellow green to straw yellow when in diapause, and are capable of changing color in either direction overnight.

A usually small emergence of fresh eurydice occurs in some localities in New York, New Jersey and Pennsylvania in the first half of August, four to five weeks after the principal emergence. Males of this late "brood" are frequently of the dark form noted above. It is very unlikely that these butterflies are descendants of those which emerged a month earlier. There may be a genetic basis for the emergence times; a bimodal emergence of Hyalophora cecropia (L.) (Saturniidae) was recently reported by Sternburg and Waldbauer (1969), with no genetic data. We do not believe the late eurydice are identical with fumosa, but the slight possibility exists that they represent another sibling species, unrecognizable in the adult except by its flight period and a statistical color difference. We have not obtained ova from these insects.

Food Plants. - Dos Passos (1969) speculates that a food plant difference between $L$. eurydice and L. appalachia is likely. However, our observations suggest that both are sedge-feeders and that neither is species- or group-specific within Carex. Female appalachia occur near sedges in shrub swamp or forest habitats where observation is difficult. One oviposition was seen in the field, on Carex lacustris Willd. (Cyperaceae) at Texas Hollow, Schuyler Co., N.Y. Other sedges commonly associated with this species in New York, all of which were completely acceptable in the laboratory, are Carex gracillima Schwein., C. lanuginosa Michx., and Scirpus georgianus Harp. Wild hosts of L. e. eurydice in central New York include C. lacustris, C. stricta Lam., C. rostrata Stokes, and C. trichocarpa Michx. All of these sedges were fully acceptable to both species, as are some dozen other species tested (mostly undetermined). We reared both species from egg to adult on Carex torta Boott. Neither species would accept any of the following grasses (Gramineae) : Festuca ovina L.; Elymus riparius L.; Brachyelytrum erectum (Schreb.) Beauv.; Muhlenbergia schreberi Gmel.; Agrostis alba L.; Phalaris arundinacea L.; Leersia oryzoides (L.) Sw.; Echinochloa crus-galli (L.) Beauv. (B. erectum and P. arundinacea are wild food plants of Lethe portlandia anthedon A. H. Clark, and $M$. schreberi is acceptable in the laboratory; Shapiro and Cardé, I970.) 


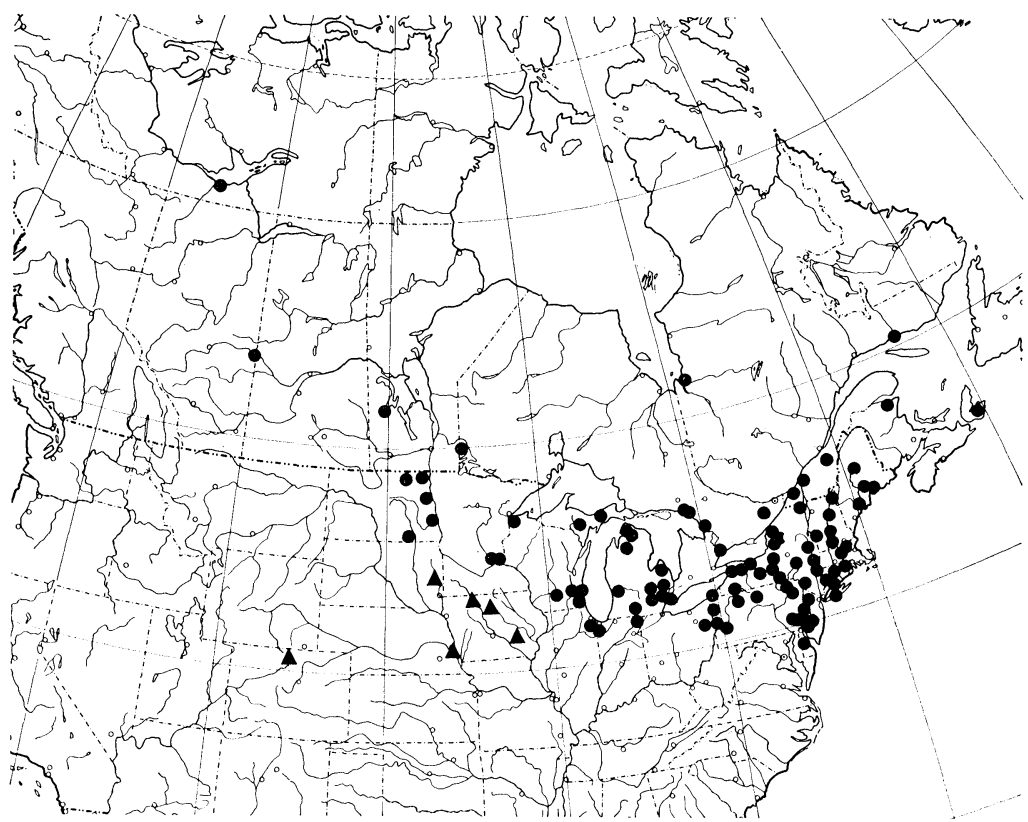

Fig. 21. Distribution of Lethe eurydice eurydice (solid circles) and Lethe eurydice fumosa (solid triangles).

Aduli Behavior. - The most striking difference between $L$. $e$. eurydice and L. appalachia, and the one leading to the discovery of their sympatry, is their differential habitat selection (Shapiro and Cardé, 1970). At the McLean Bogs Reserve, Tompkins Co., New York, these two species are frequently found flying within a few feet of each other, but do not mix. The preference of L. appalachia for shaded habitats often results in its association with $L$. p. anthedon upland or L. p. portlandia on the Coastal Plain. We have found L. e. eurydice only in relatively open sedge marshes or, rarely, in drier meadows; it never enters dense shrub swamp or woods. We have seen $L$. eurydice and L. p. antliedon in copula in their usual habitats, once each ( 3 p.m. and $3: 30$ p.m., respectively).

\section{Discussion}

Although the term "sibling species" has been in the literature for nearly thirty years and the concept is even older, it still seems necessary to point out that excessive dependence on morphological 


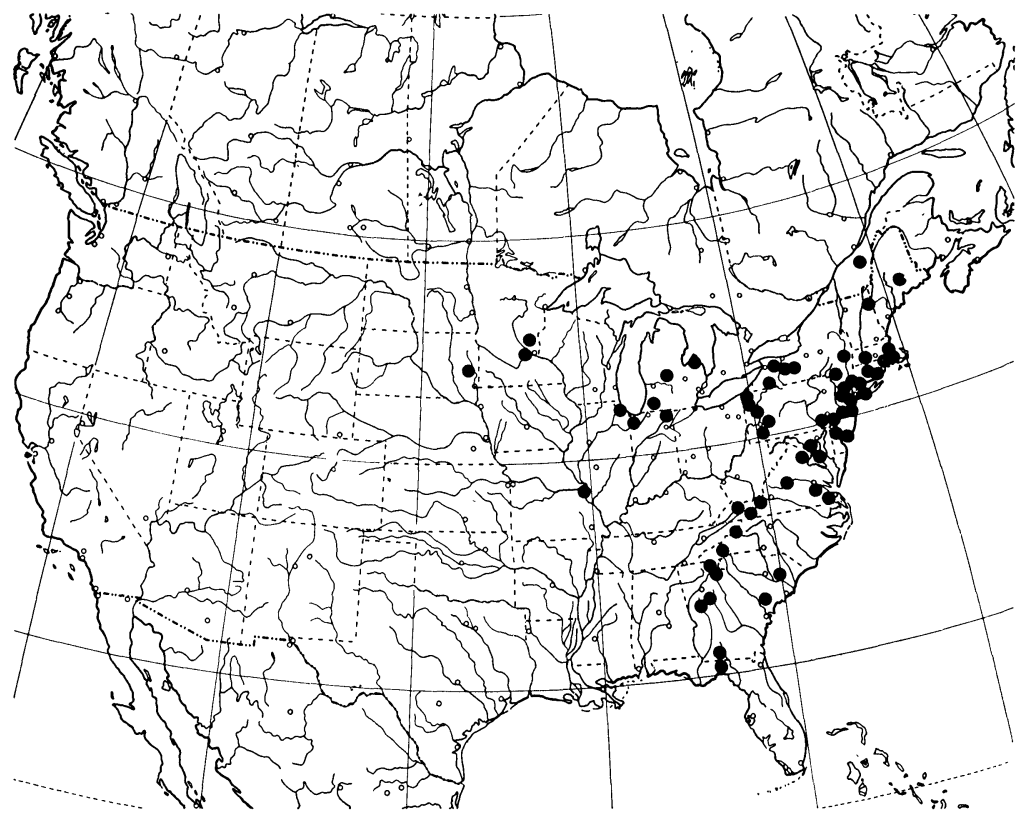

Fig. 22. Distribution of Lethe appalachia.

differences can hinder the recognition of such biologically interesting species as those of the Lethe eurydice group. Despite abundant museum evidence of sympatry, these species went unrecognized for twenty years after Chermock ( I947) was unable to find genitalic differences between them.

As usually happens with sibling species, recognition on biological grounds has led to discovery of morphological characters hitherto overlooked. These, however, are of a magnitude which would not be considered diagnostic of species in most groups of Lepidoptera. In fact, the genitalia seem to be among the most conservative characters in Lethe. Chermock found only very minor genitalic differences between $L$. portlandia and $L$. creola Skinner in the other American species group, and circumstantial evidence suggests that portlandia itself is really a pair of (largely allopatric) sibling species. Many Asiatic Lethe we have examined also show only slight differences among themselves and from their close American relatives. We consider it likely that what we are calling Lethe eurydice fumosa may also prove specifically distinct when its biology 
- particularly the early stages - becomes better known. Similar cases recently uncovered in the Lepidoptera include the tortricid moths Archips argyrospilus and A. mortuanus, which differ only in sex attractant and in some characters of the last-instar larva (Roelofs and Comeau, I969), and the papilionid butterflies Papilio zelicaon and $P$. gothica, said to differ consistently only in host-plant specificity but to behave as species in genetic tests (Remington, I968). The Holomelina aurantiaca complex (Arctiidae), often thought to consist of two species, actually includes at least ten, exceedingly similar in genitalic morphology, color and pattern, but differing in chromosome number (Cardé, unpublished).

The seemingly inevitable problem with sympatric sibling pairs such as Lethe eurydice and appalachia is to account evolutionarily for the "elegant" manner in which they coexist. The view that reproductive isolating mechanisms and ecological differences evolve in response to deleterious hybridization and competition in secondary sympatry (Brown and Wilson, I956) is now very widely accepted. It was recently challenged by Ehrlich and Raven (I969), who proposed that isolating mechanisms usually develop during the genetic differentiation of allopatric populations under different selective regimes. This is in effect a reformulation of the view of most nineteenth- and early twentienth-century evolutionists. Attempting to explain the ecological relationship of a given set of sibling species requires consideration of the following points:

I. The apparent absence of ecological interaction (e.g., competition) or gene flow between presently sympatric populations does not rule out such events in the past, nor for competition, in the future. Furthermore, intermittent large-scale gene flow between normally allopatric populations, associated with fluctuations in population sizes, has probably been an important component of speciation (Brown, I957). Such fluctuations could also result in episodes of competition between otherwise non-competing species.

2. Biogeographical evidence may offer important clues to episodes of prior sympatry or allopatry in the evolution of species differences (cf. Mengel, I964).

3. In the absence of evidence for character displacement, it cannot be assumed that biological differences which appear to prevent competition evolved in response to the adverse effects of competition.

The ecological differences among the American species of Lethe can be resolved into two parts: that involving the eurydice group alone and that concerning the eurydice and portlandia groups. The 
species eurydice, appalachia, and portlandia (in the broad sense, including anthedon) divide neatly into "non-competing" pairs: the two sedge feeders (eurydice and appalachia) differ in habitat; the two woodland species (appalachia and portlandia) differ in larval food plant. (Similarly, in sexual behavior, eurydice and appalachia are essentially non-territorial; portlandia is strongly territorial.)

Lethe and the genera closely related to it are hypothesized to have originated in southeast Asia (Miller, 1968), a region with many forest-dwelling, grass- (mostly bamboo-) feeding representatives of both the eurydice and portlandia groups. It seems reasonable that the ancestors of both these groups migrated to North America via the Bering land bridge in the Arcto-Tertiary forest, and were forced southward by the events of the Pleistocene.

With the vast majority of the many Asian Lethe feeding on grasses, the evolution of sedge feeding in North America by the ancestor of the eurydice group is a tempting hypothesis. Evolution of this trait by proto-eurydice independent of competition with proto-portlandia or by character displacement in sympatry with it are both possibilities. T. Shirozu (pers. comm.) informs us that Lethe marginalis Motschulsky, which seems to be a member of the eurydice group, feeds on non-bamboo grasses and on sedges in Japan, as does Kirinia epaminondas Staudinger, formerly placed in Lethe. Ninguta ("Lethe") schrenckii Menetries is an obligate sedge feeder.

On the other hand, the speciation of eurydice and appalachia may have occurred when one of the Pleistocene glaciations isolated some populations of proto-eurydice in prairie to the west of populations in the eastern Austral forests. Virtually the entire range of eurydice was glaciated, and the distribution is therefore of recent origin. The same can be said for the northern portions of the ranges of appalachia and portlandia, but the southern portions are characteristic of many organisms which presumably survived the Wisconsin (and earlier glaciations) in the southeast. The lack of recorded relict populations of eurydice south of Pennsylvania in the Appalachians, if not due to inadequate collecting, suggests that the species did not have a Wisconsin refugium in the forested Austral Zone of the southeast; its habitat preference and developmental rate support this interpretation. (We do know of species of Hesperiidae, e.g. Euphyes bimacula, with ranges and biologies substantially similar to L. eurydice, which have relict populations in the southeast; Shapiro, 1970b.) Its most probable refugium, then, was in glacial Transition Zone somewhere west of the Appalachians. The existence of $L$. e. fumosa also supports 
a prior western distribution for eurydice, but does not help in dating it. We have no grounds for estimating evolutionary rates in this group; all that can be said now with some confidence is that eurydice and appalachia were more likely allopatric than sympatric in the Wisconsin (and appalachia and portlandia more likely sympatric).

The critical evidence concerns character displacement. We have found no morphological character displacement in sympatric vs. allopatric populations of Lethe eurydice and L. appalachia. One of us (Clench) believes he has observed behavioral character displacement between them in Pennsylvania, within the area of general sympatry; in certain localities where only one species occurs, it appears that the habitat selection is not so rigorous as elsewhere. This needs additional study and quantification. Another geographic area also bears close investigation in this connection. Specimens of eurydice from southeastern New York (Orange, Rockland, and Westchester Counties) are somewhat anomalous, tending to vary in color and pattern (but not genitalia) toward appalachia. We have seen very few specimens from outside this small area which we would hesitate to classify to species by color and pattern. The area is completely surrounded by normal, sympatric, well-differentiated populations of both. It is thus critical to determine the ecology of these anomalous insects. Should appalachia be rare or absent, and eurydice occupying its niche at least in part, one would have a powerful argument for character displacement as the origin of the habitat difference. (There is a chance of natural hybridization due to man's extensive disturbance of Lethe habitats in southeastern New York.)

There are, then, two basic questions: Did the behavioral and food plant differences between the American eurydice and portlandia groups evolve independently, or largely as a result of competition? Did the sharp habitat selection between eurydice and appalachia in close sympatry evolve in isolation, or was it intensified by behavioral character displacement?

On the first point, any evolutionary scenario will require much more comparative data on the Asiatic species than is readily available. Only a comprehensive revision, identifying the closest relatives of the American species and comparing their biologies, will allow a convincing argument.

On the second point, field studies in areas of allopatry will be critical. It should be noted that while we suspect the eurydiceappalachia habitat difference may have evolved to prevent competition 
for larval food, it also may function, and have evolved, as a reproductive isolating mechanism. Greater knowledge of mating behavior in this group (Shapiro and Cardé, 1970), as well as more information on the southeastern New York populations, may be able to distinguish the correct hypothesis.

Our current state of knowledge does not allow us to choose between independent evolution and character displacement in accounting for the differences between the American eurydice and portlandia groups. But character displacement is an attractive hypothesis for the eurydice-appalachia habitat selection difference.

\section{Acknowledgments}

We wish to thank William D. Field of the U.S. National Museum and Dr. Alexander B. Klots of the American Museum of Natural History for their advice and cooperation; Dr. Michael Emsley for the loan of specimens of Lethe eurydice fumosa from the Philadelphia Academy of Natural Sciences; N. D. Riley of the British Museum (Natural History) and Dr. T. Nyholm of the Naturhistoriska Riksmuseum (Stockholm) for aiding in the search for the early types; Dr. Lee D. Miller for information on Euptychia spp.; Lucien Harris, Jr. and Frank Rutkowski for field notes and unpublished locality data; Edward L. Rittershausen for specimens from southeastern New York; Dr. Takashi Shirozu, Kyushu University, Japan, for data on Asiatic Lethe; and Dr. Peter A. Hyypio for determining species of sedges. Dr. John G. Franclemont of Cornell University provided invaluable assistance in the taxonomic research. Mrs. Adrienne R. Shapiro assisted in color determinations and in the preparation of the manuscript. Dr. John Burns made several helpful suggestions.

This research received partial support from National Science Foundation Grant GB 7757 (Environmental Biology) and from the Grace Griswold Memorial Illustrations Fund, Department of Entomology and Limnology, Cornell University. To both we extend our thanks.

\section{REFERENCES}

Borsduval, J. B. A. and J. Le Conte

1829. Histoire generale et iconographie des lepidopteres et des chenilles de l'Amerique Septentrionale. Paris: Librairie Roret. 228 pp., 78 pls.

Brown, W. L., JR.

1957. Centrifugal speciation. Quart. Rev. Biol. 32: 247-277. 
Brown, W. L., Jr., and E. O. WILson

1956. Character displacement. Syst. Zool. 5: 49-64.

Butler, A. G.

1868. Catalogue of diurnal lepidoptera of the family Satyridae in the collection of the British Museum. London: British Museum (Natural History). $211 \mathrm{pp}$.

1869. Catalogue of the diurnal lepidoptera described by Fabricius in the collection of the British Museum. London: British Museum (Natural History). $303 \mathrm{pp}$.

Chermosk, F. H.

1927. New forms of Lepidoptera. Bull. Brooklyn Ent. Soc. 22: 118-119. Chermock, R. L.

1947. Notes on North American Enodias (Lepidoptera). Ent. News 58: 29-35.

1949. Alabama. Season Summary. Lepid. News 2 (suppl.): 8.

Clark, A. H.

1932. The butterflies of the District of Columbia and vicinity. U. S. National Museum, Bull. 157. 337 pp.

Cramer, P.

"1781" [1779]. De Uitlandsche Kapellen ... v. 3. Amsterdam: S. J. Baalde. $176 \mathrm{pp}$.

DENTON, S. F.

1900. Moths and butterflies of the United States east of the Rocky Mountains. v.2. Boston: J. B. Millet Co. pp. 163-268.

dos Passos, C. F.

1964. A synonymic list of the nearctic Rhopalocera. Lepid. Soc. Memoir 1. 145 pp.

1969. Lethe eurydice (Johansson) and L. fumosus (Leussler): sibling species (Lepidoptera: Satyridae). J. N.Y. Ent. Soc. 77: 117-122.

EDWARDS, W. H.

1872. Synopsis of North American butterflies. Philadelphia. $51 \mathrm{pp}$.

1897. The butterflies of North America. v.3. Boston: Houghton Mifflin Co. $435 \mathrm{pp}$

Ehrlich, P. R. and P. H. Raven

1969. Differentiation of populations. Science 165:1228-1232.

FABricius, J. C.

1775. Systema entomologiac . . . Flensburgi et Lipsiae: Kortii. 832 pp.

1781. Species insectorum . . v.2. Hamburg et Kilonii: C. E. Bohn. 494 pp.

1787. Mantissa insectorum . . v.2. Hafniae: C. G. Proft. 382 pp.

1793. Entomologia systematica ... v.3. Hafniae: C. G. Proft. 349 pp. FIELD, W. L.

1936. New North American Rhopalocera. J. Ent. Zool. (Pomona College) $28: 17-26$.

Forbes, W. T. M.

1960. Lepidoptera of New York and neighboring states. v.4. Cornell Univ. Agr. Expt. Sta. Memoir 371. 188 pp.

Godart, J. B.

"1819" [1821]. in P. A. Latreille. Encyclopedie methodique . . . v.9 Paris: Agasse. 828 pp. 
Gosse, P. H.

1840. The Canadian naturalist. London: J. Van Voorst. 372 pp.

1841. List of butterflies taken at Compton, in lower Canada. Newman's Entomologist 1: 137-139.

HARRIS, T. W.

1862. A treatise on some of the insects injurious to vegetation. Boston: W. White. 640 pp.

JOHANSSON, B.

1763. in C. Linnaeus. Amoenitates academicae . . v.6. Stockholm: Laurentii Salvii. $486 \mathrm{pp}$.

KLots, A. B.

1951. A field guide to the butterflies. Boston: Houghton Mifflin Co. $349 \mathrm{pp}$.

Kornerup, A. and J. H. Wanscher

1967. Methuen handbook of colour. London: Methuen. $243 \mathrm{pp}$.

LEUSSLER, R. A.

1916. A new variety of Satyrodes canthus from Nebraska (Lep.). Ent. News 27: 99-100.

1938. An annotated list of the butterflies of Nebraska, with the description of a new species (Lepid., Rhopalocera). Ent. News 49: 213-218.

Linnaeus, C.

1767. Systema naturae . . 12th ed. v.1. Stockholm: Laurentii Salvii. pp. 533-1327.

MASTERS, J. H.

1967. Minnesota. in J. R. Heitzman. Zone V. Season Summary. Lepid. Soc. News, 15 April.

MATHER, B.

1961. Tennessee. Season Summary. Lepid. Soc. News, 1 June.

Mather, B. and K. MATher

1958. The butterflies of Mississippi. Tulane Stud. Zool. 6: 64-109.

Mengel, R. M.

1964. The probable history of species formation in some northern wood warblers (Parulidae). The Living Bird 3: 9-43.

MilleR, L. D.

1968. The higher classification, phylogeny and zoogeography of the Satyridae (Lepidoptera). Memoirs Amer. Ent. Soc. 24: 1-174.

MORRIS, J. G.

1860. Catalogue of the described Lepidoptera of North America. Smithsonian Misc. Coll. 68 pp.

1862. Synopsis of the described Lepidoptera of North America. Part I. Smithsonian Misc. Coll. 358 pp.

Price, H. F. and E. M. Shull

1969. Uncommon butterflies of northeastern Indiana. J. Lepid. Soc. 23 : 186-188.

Puckering, D. L. and R. L. Post

1960. Butterflies of North Dakota. Bull. N. Dak. Agr. Coll., Fargo.

Remington. C. L.

1968. A new sibling Papilio from the Rocky Mountains, with genetic and biological notes (Insecta, Lepidoptera). Postilla 119: 1-40. 
RIDGWAY, R.

1912. Color standards and color nomenclature. Washington: Author. 43 pp., 53 pls.

Roelofs, W. L. and A. Comeau

1969. Sex pheromone specificity: taxonomic and evolutionary aspects in Lepidoptera. Science 165: 398-400.

SCUDDER, S. H.

1889. The butterflies of the eastern United States and Canada. v.1. Cambridge, Mass.: Author. 766 pp.

Shapiro, A. M.

1970a. The butterflies of the Tinicum region. in J. McCormick. The natural features of the Tinicum Marsh, Delaware and Philadelphia Counties, Pennsylvania, with particular emphasis on the vegetation. Appendix III. In press.

1970b. The biology of Poanes viator (Lepidoptera: Hesperiidae) with notes on post-Pleistocene range changes in some American butterflies. In preparation.

Shapiro, A. M. and R. T. CARDÉ

1970. Habitat selection and competition in sibling species of Satyrid butterflies. Evolution 24: 48-54.

Sмyтне, E. A.

1890. Notes on the southern distribution of some common butterflies. Psyche 5: 347-348.

Sternburg, J. G. and G. P. Waldbauer

1969. Bimodal emergence of adult Cecropia moths under natural conditions. Ann. Ent. Soc. Amer. 62: 1422-1429.

WEYMER, G.

1907. in A. Seitz. The Macrolepidoptera of the world. v.5. Stuttgart: Kernen. 615 pp. 

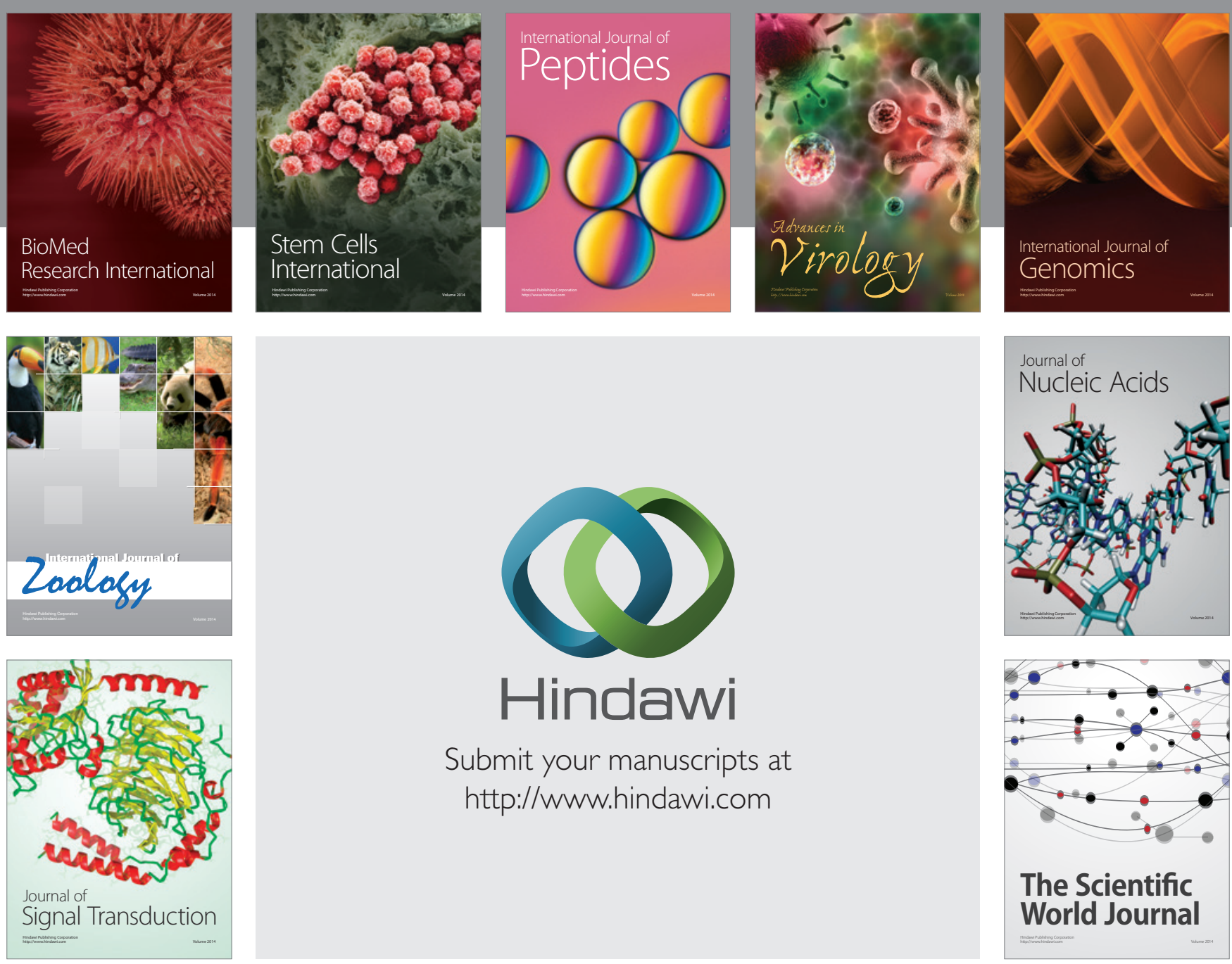

Submit your manuscripts at

http://www.hindawi.com
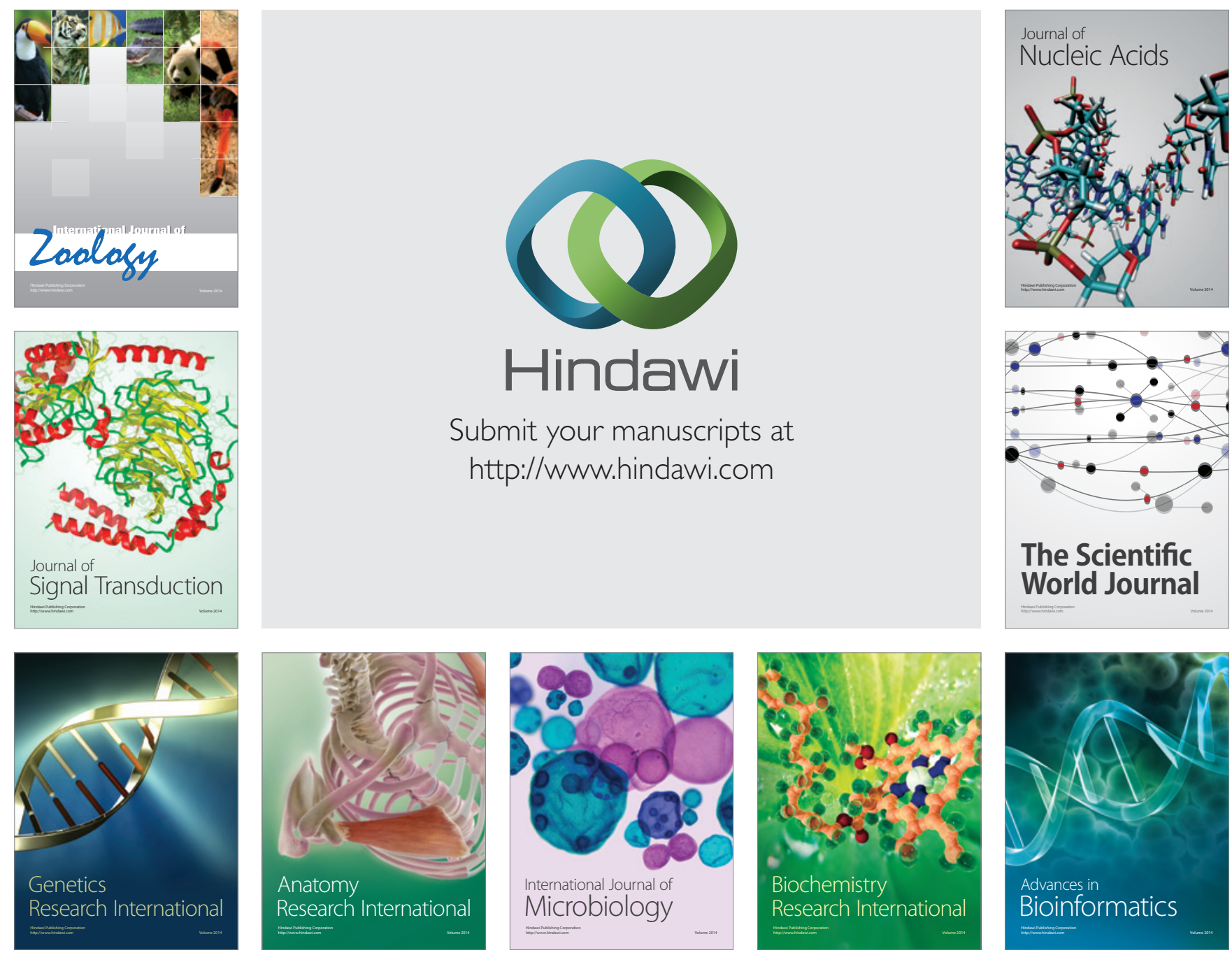

The Scientific World Journal
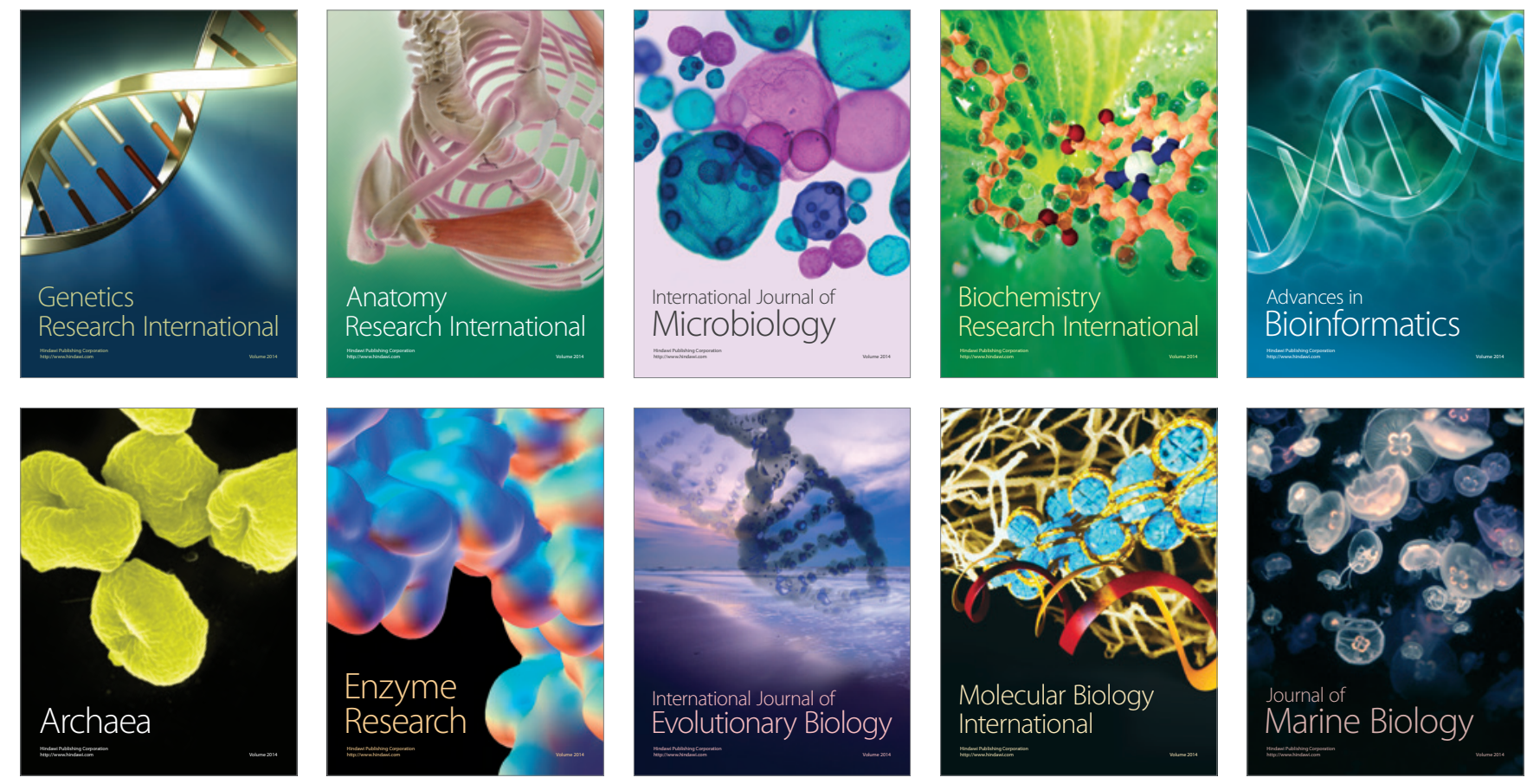